\title{
No more loose ends
}

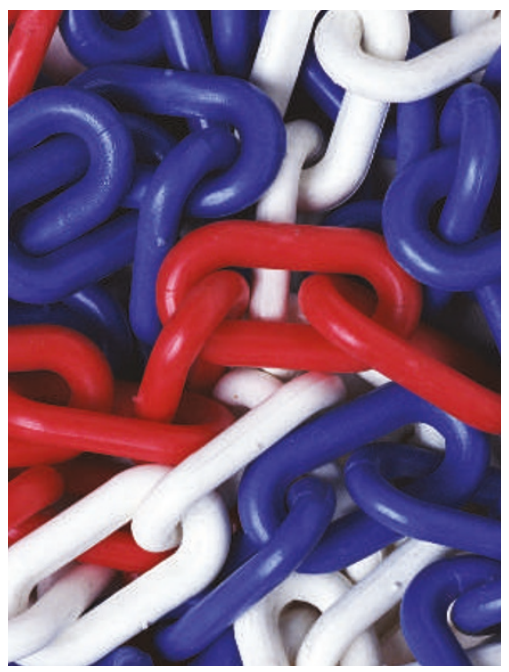

Although the cyclization of 5- and 6-membered chains is a high-yield process in synthetic chemistry, for entropic reasons, forming cyclic molecules from chains longer than 100 atoms has always been difficult; the likelihood of the ends of the molecules finding each another is low. Now, researchers in Tokyo have developed an elegant approach to the problem: by increasing the size of the ends of a molecule, the probability of them finding one another can be improved (Hori et al. Angewandte Chemie International Edition 44, 4896-4899; 2005). The authors succeeded in synthesizing an ultramacrocyclic molecule with 238 atoms in its backbone by functionalizing both ends of a long chain with large cyclic reaction centres . Instead of directly bonding, the cyclic units 'catenate' — the loops thread through one another like links in a chain. The ultramacrocycle consists of two molecules doubly catenated together: it is thought that the orientation of the reaction centres is incorrect for encouraging intramolecular catenation, and trimers or higher oligomers are entropically disfavoured. This approach could be broadly applied to overcome entropic disadvantages in other systems.

\section{ULTIMATE CLEAVAGE}

Many crystals have a layered structure with strong in-plane bonds and weaker coupling between the adjacent layers. Splitting these crystals into individual atomic layers has been so far an elusive goal. Moreover, it has been unclear whether free-standing atomic layers could exist without decomposing or segregating. A group of British and Russian researchers, who last year obtained single-layer graphene (Science 306, 666-669; 2004), now report that their method based on micromechanical cleavage (a sort of peeling) can be used to obtain atomically thin sheets of virtually any layered material (Proceedings of the National Academy of Sciences 102, 10451$10453 ; 2005)$. For instance, they successfully isolated two-dimensional crystallites of boron nitride several dichalcogenides and complex oxides. Surprisingly, these crystallites are stable over several weeks at ambient conditions, and for most of them the structural and electronic properties remain unchanged. However, for the dichalcogenide $\mathrm{NbSe}_{2}$, the two-dimensional crystal seems to behave more like a semimetal, as opposed to the normal metallic behaviour of the bulk crystal — a finding that will no doubt stimulate further investigators to look for new properties sparking from the twodimensional confinement.

\section{Micropatterns with chemical contrast}

Controlling surface energy is an increasingly important engineering parameter in a wide range of technologies and materials systems such as microfluidics microelectromechanical systems and tissue scaffolds. Although there are many conventional methods to impart tailored surface energy and chemical patterns on a variety of substrates, combinatorial approaches present practical advantages because they enable not only rapid and thorough analysis of the effect of surface chemistry over a wide range of materials systems, but also the ability to create continuous surfaceenergy gradients. Eric Amis and colleagues now report a softlithography method involving microcontact printing and vapour deposition that allows the fabrication of monochlorosilane self-assembled layers exhibiting gradual and systematic change in chemical contrast between the pattern domains (Nano Letters 5, 1535-1540; 2005).

The calibration of this chemical contrast by well-established surface-characterization techniques, such as contact-angle measurements, should prove useful as a platform for high-throughput analysis of polymer thin films, and as a reference tool for calibrating image contrast in chemically sensitive scanning probe microscopy.

\section{Use voids for strength}

Critical requirements for the effective use of bulk metallic glasses in structural applications are mechanical properties such as high strength and toughness. However, the use of these materials is currently limited by the amount of strain they can take. Wada and colleagues (Applied Physics Letters 86, 251907; 2005) are now able to significantly improve the plasticity by inducing the formation of voids. Normally, on application of strain in bulk metallic glasses, shear bands develop and lead to a reduced strain tolerance. Wada and colleagues show that the pores act as stress concentrators, resulting in a significant resilience to compressive strain without the loss of other mechanical properties such as rigidity. Although this approach has been used before, the number of pores formed meant a significant reduction in stiffness. By choosing the correct combination of pore density and size, the strength of the materials is preserved whereas the plasticity can be significantly improved, along with their potential for technical applications.

\section{Evergreen emulsions}

Many household products, toiletries and foodstuffs are based on emulsions and foams, in which a mix of oil and water is made stable by the use of emulsifiers, which orient at the interface between the two liquids. Conventionally, these emulsifiers are surfactant molecules, but there has been increasing interest in using particles because of their high free-energy of adsorption to interfaces. This means that they are effectively permanently adsorbed, resulting in longterm stability of the systems. Most emulsifiers are synthetic, but now, Bernie Binks and colleagues report the use of a naturally occurring particle, the spore of the evergreen club moss, Lycopodium clavatum (Langmuirhttp:// dx.doi.org/10.1021/ la513858). The authors found that these spores - average diameter $31 \mu \mathrm{m}$ (see figure) — preferentially form oil-in-water emulsions, and are efficient for oils of different polarity. Although the particles gave low coverage of the interface, oil droplets several millimetres in diameter were found to be indefinitely stable to coalescence. The benefit of using these spores is not only because of their efficiency, but also because they are environmentally friendly, and what's more are digestible and have a long history of use in herbal remedies. They are therefore attracting great interest for use in foodstuffs and other products. 